\title{
Factores que influyen en la gerencia de las mujeres: el caso de las pequeñas y medianas empresas colombianas
}

\section{Factors that influence the management of women: The case of colombian small and medium enterprises}

\author{
Laura Salas Arbeláez*, Mónica García Solarte, Jorge Hirs Garzón \\ Universidad del Valle, Colombia
}

Recibido el 4 de septiembre de 2018; aceptado el 22 de enero de 2019

Disponible en Internet el: 24 de enero de 2019

\section{Resumen}

El género en las organizaciones es una temática que ha inquietado a varios académicos a través del tiempo. La brecha entre hombres y mujeres al ocupar cargos directivos cada vez es menor; sin embargo, aún existen obstáculos para que las mujeres ocupen este tipo de cargos. El objetivo de esta investigación fue identificar los factores que influyen en la probabilidad que una mujer ocupe cargos gerenciales en las Pequeñas y Medianas Empresas Colombianas. Para lograr este objetivo se utilizó un enfoque cuantitativo donde se aplicaron 354 encuestas a gerentes de las Pymes. Como resultado se obtiene que los años de funcionamiento, junta directiva, junta directiva mujer, participación mujeres, sector primario y rendimiento en Sistema Abierto indican que, en la medida en que sus valores incrementan, así mismo se eleva la probabilidad de que una mujer sea gerente. Por el contrario, variables como las exportaciones, el rendimiento en relaciones humanas y racional disminuyen la probabilidad de que una mujer llegue a ocupar cargos gerenciales. Estos resultados abren las puertas a la posibilidad de mejorar las condiciones de las empresas para posibilitar que las mujeres ocupen cargos gerenciales.

\footnotetext{
*Autor para correspondencia

Correo electrónico salas.laura@correounivalle.edu.co (1. Salas Arbelaez).

La revisión por pares es responsabilidad de la Universidad Nacional Autónoma de México. 
Código JEL: J16, L20, M10

Palabras clave: Género del gerente; Pymes; Rendimiento; Junta Directiva

\begin{abstract}
Gender in organizations is a topic that has disquiet several academics over time. The gap between men and women when occupying managerial positions each time is smaller. However, there are still obstacles for women to occupy this type of positions. Thus, the objective of this research was to identify the factors that influence the probability that a woman occupies managerial positions in the Small and Medium Colombian Companies. To achieve this objective, a quantitative approach was used, where 354 surveys were applied to managers of SMEs. As a result, the years of operation, the board of directors, the board of directors, women, women's participation, primary sector and Open System Performance indicate that, as their values increase, the probability of a woman being a manager increases as well. On the contrary, variables such as exports, performance in human relations and rational decrease the probability that a woman get to occupy managerial positions. These results open the doors to the possibility of improving the conditions of companies to enable women to occupy management positions.
\end{abstract}

JEL codes: J16, L20, M10

Keywords: Manager's gender; SMEs; Performance; Board of directors

\title{
Introducción
}

Las investigaciones sobre las barreras que enfrentan las mujeres para ascender en las organizaciones y ocupar cargos estratégicos ha sido un tema de interés particular en los asuntos de género y en la perspectiva del "techo de cristal". Los estudios sobre ascensos de las mujeres a cargos directivos se han puesto en relieve en los últimos años en países europeos, estadounidenses y asiáticos (Brady, Isaacs, Reeves, Burroway, \& Reynolds, 2011; Smith, Smith, \& Verner, 2013). A pesar de que muchas mujeres han tenido acceso a la educación, al igual que sus homólogos masculinos, y han trabajado activamente, las posibilidades de acceso no han sido las mismas (Haslam \& Ryan, 2008)with particular emphasis on the role played by the so-called glass ceiling. However, recent archival evidence has identified an additional hurdle that women must often overcome once they are in leadership positions: the glass cliff [Ryan, M. K., \& Haslam, S. A. (2005a. Algunos autores (Hurley \& Choudhary, 2016; Smith et al., 2013) han atribuido la escasez de mujeres en cargos directivos a elementos como el número de hijos, estado civil y licencias de maternidad; otros por su parte se han ido por características más asociadas a las incompatibilidades percibidas entre las habilidades de las mujeres y los requisitos de liderazgo que exige un cargo de dirección (Cárdenas et al., 2014; Eagly \& Carli, 2007; Eagly \& Karau, 2002). Hurley \& Choudhary (2016) señalan que 
ocupar un cargo de alta dirección, ya sea que lo haga un hombre o una mujer, requiere una combinación de factores individuales y de nivel medio.

Desde los 2000, autores como Fawcett \& Pringle señalan que la incompatibilidad de las mujeres en cargos directivos y la escasez de ellas como Chief Executive Officers (CEOs) hace que estas utilicen características femeninas y masculinas para alcanzar estos puestos de alta dirección. Para Glass \& Cook (2016) las mujeres en cargos directivos tienen un enorme potencial para contribuir positivamente a las organizaciones; además, la representación de las mujeres reduce la segregación de género en las organizaciones. Se entienden desde cualquier ángulo que factores que dan forma a la experiencia y el éxito de las mujeres van más allá de las barreras creadas por el "techo de cristal". Los determinantes para que una mujer ocupe un cargo directivo son variados; sin embargo, aunque estas investigaciones han sido diversas aún no se ha podido reducir la brecha que existe entre hombres y mujeres para ocupar este tipo de cargos.

En los últimos años a nivel mundial, la participación de las mujeres en el mercado laboral se ha mantenido estable, situándose en el 52\%. Por el contrario, las tasas de participación en la fuerza laboral a nivel mundial para los hombres han disminuido de forma constante del $81 \%$ al 77\% (Foro Económico Mundial, 2017). En este sentido y tal como lo menciona el Centre for Social Development, Humanitarian Affairs, UNICEF, \& United Nations Development Fund for Women (2015), las brechas de género cada día son menores y con el tiempo las mujeres han empezado a ocupar posiciones tradicionalmente ocupadas por hombres.

Según el informe del Foro Económico Mundial (2017), la diferencia de participación de hombres y mujeres a nivel mundial para el 2017 situó a Colombia en el puesto 36 entre 144 países analizados frente a la brecha de género, lo cual indica que el país se encuentra dentro de un nivel en el que el hombre y la mujer tienen participación similar en ámbitos económicos, políticos, educativos y de la salud. Sin embargo, en la ocupación de cargos gerenciales solo el $11,5 \%$ de las organizaciones son dirigidas por mujeres. Ahora bien, en el contexto latinoamericano y especialmente colombiano, las características que explican las probabilidades de que una mujer ocupe un cargo gerencial son escasos. Se sabe con antelación que hay elementos de orden individual pero también de orden organizacional que pueden afectar que una mujer ocupe o no un cargo de este nivel.

Por su parte, las pequeñas y medianas empresas se han convertido en un foco de estudio debido a la gran participación que tiene en contexto latinoamericano. Estas cumplen un papel clave en la economía mundial y más en la de cada país (Di Ciano, 2010). La mayor parte de los países del mundo por lo general tienen entre el $50 \%$ y el $90 \%$ de los empleados en este grupo de empresas y por ello resalta que este tipo de empresas son el motor de cualquier economía. Un país con más empresarios locales será más rico y equitativo, por lo tanto es fundamental estudiarlas y conocer su comportamiento y sus estrategias. De acuerdo a esto, 
Valencia (2000) señala que las pequeñas y medianas empresas son consideradas como el sector productivo de mayor importancia en muchas economías, tanto en los países desarrollados como en los emergentes. En el caso de Colombia, el 86\% de las empresas (entre la grande, pequeña y mediana empresa) son pequeñas y medianas (Cámara de Comercio, 2014), lo cual hace que esta investigación se focalice en esta unidad de análisis.

Por lo tanto, el objetivo de esta investigación es identificar los factores que influyen en la probabilidad de que una mujer ocupe cargos gerenciales en las Pequeñas y Medianas Empresas (Pymes) en Colombia. El Ministerio de Comercio, Industria y Turismo (2012) y el Congreso de la República de Colombia (2004) por medio de la Ley 905 decreta como Pymes a las pequeñas y medianas empresas, unidades de utilización económica, que se forman por personas naturales o jurídicas, dedicadas a actividades empresariales, agropecuarias, industriales, comerciales o de servicios, rural o urbana, que cumpla con unos requisitos establecidos: Pequeña Empresa: personal entre 11 y 50 trabajadores. Activos totales mayores a 501 y menores a 5.001 salarios mínimos mensuales legales vigentes. Mediana: personal entre 51 y 200 trabajadores. Activos totales entre 5.001 y 15.000 salarios mínimos mensuales legales vigentes.

Dentro de las variables que afectan el comportamiento de las Pymes se consideran a nivel individual, la edad y el nivel de estudios y dentro de las variables organizacionales se encuentra la junta directiva, la participación de las mujeres en la junta, la participación de las mujeres en número de empleados, años de la empresa, sector, ventas, mercado extranjero y rendimiento.

Esta investigación en su primera parte muestra la revisión teórica de los conceptos y variables que se abordan. En la segunda parte señala la metodología con la muestra utilizada, la definición de las variables y la validez de la misma. Igualmente se muestra el modelo utilizado. En la tercera parte se muestran los resultados descriptivos y univariantes para finalmente dar las conclusiones principales de la investigación y la aceptación o no de las hipótesis.

\section{Revisión de la literatura}

\section{Género}

El género es un concepto abordado desde distintos puntos de vista y, así mismo, definido de diversas maneras según el contexto (social, cultural, político, económico) con el que conviven quienes construyen dicho concepto. Para comenzar, el origen de la caracterización entre hombre y mujer conforma un hecho primario de la vida simbólica y de la comunicación en el eje central de la sociedad. La primera utilidad del concepto de género es determinar que hombres y mujeres tienes más diferencias que similitudes, es así como la sociedad humana en general reconoció la existencia de estos dos géneros (Jayme \& Sau, 2004).

La concepción del género masculino y femenino, a partir de fundamentos biológicos, sociales y culturales, implica algunas diferencias relevantes en el mundo organizacional, es- 
pecialmente en lo que se refiere a comunicación interna y el liderazgo. Desde la concepción biológica, el género se configura y nace a partir de la determinación cromosómica del sexo. Al estudiar el género dentro de las organizaciones, desde la anterior perspectiva, Powell \& Eddleston (2011) aclaran que hombres y mujeres desarrollan y gerencian sus negocios de maneras diferentes, sugiriendo que el sector de la organización, su tamaño y edad, la cultura organizacional, el liderazgo, su nivel de innovación y el desempeño, se configuran como herramientas de caracterización que conllevan a establecer diferencias de gestión desde cargos gerenciales. El conocimiento y aprovechamiento de estas diferencias ayudan a establecer mejores políticas en la organización, tomar mejores decisiones, y con base a una estructuración de diversidad de género en la alta gerencia, lograr mejores resultados en los ámbitos anteriormente mencionados, donde adicionalmente para cierto tipo de organizaciones se puede obtener un mejor apoyo, a la vez que ejercer un mejor control de parte de la alta gerencia al gerente (Adams \& Ferreira, 2008).

En las discusiones de género, se introduce el concepto de techo de Cristal (Burin,2008)el cual aparece en la incorporación de la mujer al mercado laboral. El primer planteamiento se realizó en 1987 en el libro Breaking the Glass Ceiling: Can Women Reach the Top of America's Largest Corporations? En este texto, los autores revelaron cómo el ambiente ejecutivo para las mujeres es diferente al de los hombres, pues deben enfrentarse a obstáculos en el camino para alcanzar cargos de alta gerencia. El techo de cristal es la existencia de una barrera transparente que les impidiera acceder o ascender por mecanismos no siempre visibles de discriminación. El techo de cristal es entonces un freno en la carrera profesional de la mujer, aun cuando esta haya logrado tener un alto desempeño y una adecuada formación profesional. (Barnet-Verzat \& Wolff, 2008; Freeman, 1990; Meza Martínez, 2018).

\section{Género y Estudios académicos}

Oakley (2000) señala que son pocas las mujeres en la alta gerencia que tienen experiencia con varias líneas de sucesión. Para ello, este autor realiza una encuesta a 461 ejecutivas senior y 325 CEOs de las empresas más grandes de Estados Unidos, donde encontraron que los estudios de estas mujeres se enfocaban principalmente para trabajar en áreas de recursos humanos y relaciones públicas, de modo que, para ser mujeres gerentes y ser promovidas, estas deberían tener mayor nivel de estudios en estas áreas. Por otro lado, Hurley \& Choudhary (2016) y Hodigere \& Bilimoria (2015) indican que cada año adicional de educación reduce las probabilidades de tener una mujer CEO. Por lo tanto, en comparación con los hombres, tener mayor educación no aumentará la probabilidad de las mujeres lideren una organización. Por su parte, Burgess \& Tharenou (2002) plantean que los análisis de los niveles educativos son difíciles debido a las diferencias de educación en los países. Por lo anterior, se plantea: 
H1: Las mujeres con mayor nivel educativo tienen mayor probabilidad de ser gerentes en una Pyme.

\section{Género y Edad}

Withisuphakorn \& Jiraporn (2017)we study CEO gender and CEO age. Because women face significantly more obstacles in advancing their careers, it may take them longer to reach the top position, i.e. the chief executive officer (CEO estudian el género del gerente y la edad de los mismos. Como resultado de esta investigación, los autores encuentran que a causa de que las mujeres tienen más obstáculos para avanzar en sus carreras, se demoran más en liderar una empresa. Así, las mujeres gerentes deberían ser de mayor edad que sus contrapartes hombres. No obstante, los autores concluyen que las mujeres son más jóvenes, aunque con una diferencia media de dos años en un promedio de 50 años de edad. Elsaid (2015) y Hodigere \& Bilimoria (2015) llegan a la misma conclusión señalando específicamente que ser más joven aumentará la probabilidad de las mujeres para ocupar un cargo directivo. Por lo anterior, se plantea:

H2: Ser más joven aumentará la probabilidad de las mujeres de ser gerente en una Pyme.

\section{Género y años de la empresa}

Ali \& Shabir (2017) señalan que las empresas que son lideradas por mujer son empresas generalmente maduras, es decir, tienen más de 20 años de constitución. Los autores indican que, a medida que una mujer ocupa un puesto de gerencia en las organizaciones, los años de la empresa también aumentan. Ahora bien, Araújo-Pinzón, Álvarez-Dardet, Ramón-Jerónimo \& Flórez-López (2017) argumentan que las mujeres tienen un tiempo de promoción significativamente menor que sus homólogos, por lo tanto esto hace que las mujeres trabajen en empresas más jóvenes y que al mismo tiempo la empresa tenga menos posibilidad de exportación.

Por su parte, Díaz \& Jiménez (2010) encuentran que las empresas dirigidas por mujeres afrontan ciertas desventajas estructurales, lo que las lleva también a liderar empresas más jóvenes. Las desventajas que estos autores señalan están enfocadas en la poca disponibilidad de capital humano, social y financiero. Por otro lado, Gupta \& Raman (2014) indican que la antigüedad de la empresa no se ve afectada por tener una mujer liderando la empresa. Por lo anterior, se plantea:

H3: Las Pymes gestionadas por mujeres son más jóvenes.

\section{Género, Junta Directiva, participación de mujeres en la junta}

Adams \& Ferreira (2009) señalan que las empresas que tienen mujeres en la dirección tienen juntas más grandes que las empresas dirigidas por hombres. Cook \& Glass (2014) 
señalan que la participación de la mujer en la junta directiva no aumentará la probabilidad de que una mujer sea nombrada como gerente. Por su parte, $\mathrm{Ng} \&$ Sears (2017) realizan un estudio en 278 empresas donde analizan los determinantes organizacionales a nivel macro de las mujeres en la dirección de una empresa. Los resultados de esta investigación indican que la presencia de mujeres gerentes se asocia positivamente con el porcentaje de mujeres en la junta directiva, aspecto que también encuentran Smith et al. (2013) dentro de su investigación. Gupta \& Raman (2014) y Matsa, Miller, \& Bertrand (2011) indican que después de tener en cuenta la influencia del entorno favorable a las mujeres en el que opera la empresa, existe una asociación positiva y significativa entre la proporción de mujeres en la junta directiva y la probabilidad de que la gerente sea una mujer. Por lo anterior, se plantea:

H4: El tamaño de la junta directiva es mayor cuando el gerente es mujer.

H5: En las Pymes gestionadas por mujeres hay una mayor participación de mujeres en la junta directiva.

\section{Género y participación de mujeres}

Smith et al. (2013) señalan que cuando la empresa cuenta con una mujer como gerente existen mayores oportunidades de que la participación de la mujer aumente en la organización y especialmente en cargos de gerencia media. Dentro de los argumentos que señalan los autores se encuentra la eliminación de los prejuicios contra las mujeres o una mejor recepción de las características de las personas que desean trabajar en la organización y llegan como candidatas. Igualmente, se atribuye el hecho de cambio de percepción de los hombres por tener esposa e hijos. Del mismo modo, Matsa et al. (2011) señalan que una mujer gerente puede aumentar el deseo de contratar mujeres en la organización, por lo tanto aumenta la participación de las mismas. De lo anterior se plantea:

H6: La participación de las mujeres en las Pymes aumenta cuando la empresa es gestionada por una mujer.

\section{Género y Sector}

Kirsch (2017) pone en relieve la importancia reconocer los factores que influyen en la probabilidad de que una mujer pueda ser gerente, dentro de los cuales se encuentra el sector. Para Brady et al. (2011) el sector es la característica de la empresa estudiada con mayor frecuencia en los análisis de mujeres ejecutivas y gerentes. Dentro de la investigación de estos autores, es menos probable que las mujeres gerentes estén en sectores de la construcción, pero es más probable que estén presentes en el sector comercio. Por su parte, Matsa, Miller, \& Bertrand (2011) señalan que las mujeres tienen habilidades específicas que son más valiosas en algunos entornos, como la comercialización. García, García Pérez de Lema \& Madrid Guijarro (2012) señalan que las empresas gestionadas por mujeres se encuentran en 
el sector de servicios, y este aspecto aumenta la probabilidad de que la mujer ocupe un cargo directivo. Por lo anterior, se plantea:

H7: Las empresas que pertenecen al sector terciario son gestionadas por mujeres.

\section{Género y ventas}

Dentro de las investigaciones de Brady et al.(2011) se identifica que las empresas con mayor crecimiento de ventas tienen menos probabilidades de tener mujeres gerentes. Por su parte, Ali \& Shabir (2017) señalan que el crecimiento anual de las ventas en las empresas lideradas por mujeres es comparativamente mayor que en las empresas lideradas por hombres. Se han realizado diversos estudios que indican de qué manera puede afectar el género (hombre o mujer) en el nivel de ventas. En este sentido, Hoogendoorn, Hessel y Mirjam (2013) estimaron el impacto de la participación de las mujeres en el desempeño de los equipos de negocios. De acuerdo con los resultados, en los equipos en los que hay una proporción igual de hombres y mujeres los resultados son mejores que en los equipos en los cuales predomina algún género.

Por otra parte, Duarte \& García (2017) realizaron un trabajo cuyo objetivo fue determinar si existen diferencias en la competitividad de las empresas dirigidas por hombres y mujeres, medidas por el nivel de ventas. Los autores concluyeron que no existe diferencia entre el nivel de ventas de las empresas dirigidas por hombres y mujeres, aunque muchos estudios antecedentes señalan que son menores los rendimientos de las empresas dirigidas por mujeres que los dirigidos por hombres. De la misma manera, Beldad, Hegner y Hoppen (2016) estudiaron si el género del asesor de ventas tenía una influencia positiva en la creencia de los clientes, la confianza y la intención de compra; acorde con los resultados obtenidos, no existen diferencias significativas de acuerdo con el género del vendedor. Por lo anterior, se plantea: H8: El crecimiento de las ventas en empresas gestionadas por mujeres es menor.

\section{Género y Mercado extranjero}

En cuanto a la repercusión del género del gerente y su incidencia en el desempeño exportador de las empresas, Lee, Paik y Uygur (2016) y Welch, Welch \& Hewerdine (2008) lograron determinar que las empresas tanto grandes como Pymes de propiedad masculina logran mejores resultados de exportación. Lo anterior debido a la correcta utilización de las capacidades de mediación como lo son el marketing y la innovación. Además, estos autores proporcionaron nuevas perspectivas sobre la influencia del género en la exportación y el comportamiento empresarial y encontraron que las empresas propiedad de mujeres tienen menos probabilidad de exportar frente a las que son propiedad de hombres, esto debido a que esta práctica está asociada a características que van ligadas más al género masculino. Sin embargo, las que tuvieron éxito exportando lo describieron como una experiencia que cambió sus vidas y les permitió crecer personalmente y tener grandes resultados dentro de su empresa. 
García y Moreno (2009) mostraron también que las empresas dirigidas por mujeres sufren ciertas desventajas estructurales, una de estas y de gran importancia es la baja disponibilidad de capital humano con el que suelen contar, factor que genera cierta desventaja en la incursión en mercados internacionales. Por otra parte, Williams (2013) encontró que las empresas propiedad de mujeres son pequeñas y de menor antigüedad, y como los resultados de edad empresarial y tamaño son importantes a la hora de involucrarse exitosamente en operaciones internacionales, tienden a tener mayores dificultades.

H9: En las empresas gestionadas por mujeres la participación en el mercado extranjero es menor con respecto a la de los hombres.

\section{Género y Rendimiento}

Adams \& Ferreira (2009) señalan que las empresas que tienen mujeres en la dirección tienen un peor desempeño en términos de ROA. Haslam \& Ryan (2008) señalan el “fenómeno por el cual las mujeres son más propensas que los hombres a ser designadas para puestos de liderazgo asociados con un mayor riesgo de fracaso" (p.2). Los resultados de estos autores señalan que la selección de una mujer antes que un hombre igualmente calificado aumenta cuando el rendimiento de la organización disminuye en lugar de mejorar. Dentro de las razones que estos autores destacan se encuentra la particularidad de la mujer para manejar situaciones estresantes en su posición de liderazgo. Por lo tanto, los autores señalan que las mujeres son seleccionadas preferentemente para posiciones de liderazgo cuando una empresa se encuentra en crisis.

Para Adams, Gupta, \& Leeth (2009), Elsaid (2015), Acar (2015), Glass \& Cook (2016), Elsaid \& Ursel (2017), las mujeres en posiciones directivas están más dispuestas a asumir riesgos que los hombres, por lo que coinciden con el hecho de ocupar estos lugares cuando la empresa se encuentra en una posición de rendimiento financiero débil. Igualmente, Gupta \& Raman (2014) señalan que las mujeres gerentes son más propensas a ser designadas en empresas que son menos rentables y tienen un mayor apalancamiento. Ryan \& Haslam (2005) revelan que durante un periodo de declive general del mercado de valores, las empresas prefieren a las mujeres para sus juntas directivas y en los cargos de dirección en general. Para los autores, estos resultados señalan un obstáculo adicional que las mujeres deben superar en el lugar de trabajo.

Por su parte, Eduardo \& Poole (2016) muestra que el rendimiento no es un factor determinante para que una mujer ocupe un cargo de gerente. Ali \& Shabir (2017) coinciden con estos autores señalando que no hay diferencia en el rendimiento cuando una empresa es liderada por un hombre o una mujer, sin embargo señalan que estos aspectos varían considerablemente según los parámetros de rendimiento comercial del crecimiento anual de las ventas y la utilización de la capacidad. 
En el contexto colombiano, Moreno-Gómez \& Calleja-Blanco (2018) con propósito de analizar la relación entre la presencia de mujeres en cargos corporativos y el desempeño financiero de la empresa concluyeron que las mujeres ocupan cargos directivos en una empresa por el interés de mejorar la reputación, inspirar y mejorar las relaciones con los empleados, mantener buenas relaciones con las consumidoras y explotar las ventajas del liderazgo. Por lo tanto, estos factores aumentan la probabilidad de la presencia de una mujer gerente. De esto se plantea:

H10: En la medida en que aumenta el rendimiento de la organización disminuye la probabilidad de que una mujer sea gerente.

\section{Metodología}

La metodología de esta investigación es de enfoque cuantitativo y de tipo explicativa. Con el fin de estudiar los factores que influyen en la probabilidad que una mujer ocupe cargos gerenciales en las compañías, se realiza una encuesta a través de muestreo probabilístico aleatorio de pequeñas y medianas empresas de todos los sectores económicos en la ciudad de Cali (Colombia) durante el año 2016. La muestra incluye 354 empresas elegidas de manera aleatoria del total de 7.068 compañías registradas. Para el cálculo de la muestra se utiliza un margen de error del 5\% y un nivel de confianza del 95\%. Del total de la muestra, $260(73,4 \%)$ son dirigidas por hombres y $94(26,6 \%)$ son dirigidas por mujeres.

\section{Variable dependiente}

- Género del gerente: El término género significa ser hombre o mujer. Para esta investigación la variable es dicotómica y toma el valor de 1 si el cargo del gerente es ocupado por una mujer y 0 en caso contrario.

\section{Variables independientes}

- Edad del gerente: En esta investigación la edad del gerente es una variable continua que indica el número de años del gerente.

- Nivel educativo del gerente: Variable categórica que toma el valor de 0 para los gerentes con nivel educativo igual a bachillerato y 1 para los gerentes con un nivel educativo superior a bachillerato.

- Años de la empresa: Número de años que lleva funcionando la empresa desde su fundación.

- Proporción de mujeres en la junta directiva: Porcentaje de mujeres en la junta directiva con respecto al número total de miembros de la junta.

- Participación de mujeres: Proporción de trabajadoras mujeres en la empresa con 
respecto al total de empleados.

- Log Ventas: Representa el logaritmo natural del total de ventas de la empresa para el año 2014.

- Número de socios: Número total de socios de la empresa.

- Mercado extranjero: Variable categórica que es igual a 1 si la empresa realiza ventas en el exterior y 0 en cualquier otro caso.

- Sector Económico: Variable categórica que toma el valor de 0 si la empresa no pertenece al sector y 1 si pertenece.

- Rendimiento: El rendimiento se mide a través de cuatro modelos: Modelo de los Procesos Internos, Modelo de Sistema Abierto, Modelo Racional y Modelo de Relaciones Humanas (Quinn \& Rohrbaugh, 1983). Se elaboran índices a partir de los promedios de los valores asignados a las doce preguntas de la encuesta de la sección de rendimiento que toman valores entre 1 y 7 . Adicionalmente se calcula el índice de Rendimiento general promediando los valores de los cuatro modelos anteriores.

Para evaluar la fiabilidad del concepto de Rendimiento a través de las subvariables, se realiza un análisis de fiabilidad con alfa de Cronbach que da como resultado 0,90, un resultado favorable para el instrumento. Para los 4 modelos de rendimiento se obtuvieron los siguientes resultados en las cargas factoriales: Modelo de los Procesos Internos $(0,917)$, Modelo de Sistema Abierto $(0,868)$, Modelo Racional $(0,866)$ y Modelo de Relaciones Humanas $(0,860)$.

\section{Procedimiento de la investigación}

La primera etapa de la investigación consistió en realizar una revisión teórica de todos los conceptos a trabajar. Dentro de esta revisión se eligieron los modelos de cada concepto para incorporar en el instrumento. En la segunda etapa se diseña la encuesta como técnica de recolección, la cual fue validada teóricamente y por expertos del área de conocimiento. La aplicación de la encuesta se hizo de manera presencial visitando a cada uno de 354 los gerentes de la base de datos con la que se contaba. En la tercera etapa, se tabulan los datos y procesa la información a través del programa SPSS. El modelo con el cual se procesa la información es el modelo Logit en donde se introducen en el software la variable dependiente e independientes. Con los resultados obtenidos, se analizan los datos y se cruzan los resultados.

\section{Resultados y discusión}

En esta sección se presentan los resultados del modelo y los estadísticos descriptivos. Para empezar, en la Tabla 1 se muestran los estadísticos descriptivos de las empresas según 
el género del gerente. El T-test de diferencia de medias muestra que, con respecto a los hombres, las mujeres dirigen empresas con más años de antigüedad, cuando son gerentes hay una mayor proporción de mujeres en la junta, tienen un mayor número de trabajadoras mujeres, las ventas son menores y con rendimiento en relaciones, racional y procesos más bajo.

Tabla 1

Estadísticos descriptivos de las Pymes según el género del gerente

\begin{tabular}{|c|c|c|c|c|c|c|}
\hline \multirow[b]{2}{*}{ Variables } & \multicolumn{2}{|c|}{ Media } & \multicolumn{2}{|c|}{ Desviación Estándar } & \multirow[b]{2}{*}{$\begin{array}{c}\text { T estadís- } \\
\text { tico }\end{array}$} & \multirow[b]{2}{*}{ P-value } \\
\hline & $\begin{array}{l}\text { Mujer } \\
\text { gerente }\end{array}$ & $\begin{array}{l}\text { Hombre } \\
\text { gerente }\end{array}$ & $\begin{array}{l}\text { Mujer } \\
\text { gerente }\end{array}$ & $\begin{array}{l}\text { Hombre } \\
\text { gerente }\end{array}$ & & \\
\hline Edad gerente & 60,23 & 61,86 & 11,61 & 12,58 & $-1,095$ & 0,274 \\
\hline Años de la empresa & 41,34 & 39,02 & 11,82 & 8,88 & $1,983^{* *}$ & 0,048 \\
\hline Junta mujeres & 52,93 & 30,81 & 35,16 & 25,24 & $6,517 *$ & 0,000 \\
\hline Participación Mujeres 2014 & 33,38 & 28,88 & 14,33 & 14,23 & $2,606^{* *}$ & 0,010 \\
\hline Log Ventas & 20,52 & 20,93 & 1,60 & 1,60 & $-2,043 * *$ & 0,042 \\
\hline Número de socios & 3,80 & 3,52 & 4,00 & 2,81 & 0,731 & 0,465 \\
\hline Rendimiento relaciones & 6,13 & 6433,00 & 1,03 & 0,75 & $-2,982 *$ & 0,003 \\
\hline Rendimiento racional & 6,25 & 6,46 & 0,86 & 0,74 & $-2,253 * *$ & 0,025 \\
\hline Rendimiento sistemas & 6,30 & 6,38 & 0,83 & 0,81 & $-0,868$ & 0,386 \\
\hline Rendimiento procesos & 6,19 & 6,41 & 0,91 & 0,72 & $-2,378 * *$ & 0,018 \\
\hline Nota: $(*): \mathrm{p}<0.01 ;(* *): \mathrm{p}<0.05 ;$ & ): $<<0.1$ & & & & & \\
\hline
\end{tabular}

Fuente: Elaboración propia

Por otra parte, en la Tabla 2 se presentan las características del gerente y la empresa según el género del directivo. En esta pueden apreciarse atributos tales como: nivel educativo, la presencia de junta directiva, la participación en el mercado extranjero y el sector al cual pertenece. De acuerdo con los resultados, existe una mayor proporción de mujeres con estudios superiores al bachillerato, sin embargo esta proporción es mucho menor a la de los hombres. Con respecto a la junta directiva, la mayor parte de las empresas gerenciadas por mujeres no tienen junta directiva y no participan en el mercado extranjero. En relación al sector económico, la mayor parte de las mujeres se encuentran en el sector terciario al igual que los hombres. 
Tabla 2

Características del gerente y la empresa según el género del gerente

Frecuencia según género del gerente

$\begin{array}{llll}\text { Variable } & \text { Mujer } & \text { Hombre } & \text { Total }\end{array}$

Estudios del gerente

Bachillerato

20

58

78

Superior al Bachillerato

74

202

276

Junta Directiva

$\begin{array}{cccc}\text { Sí } & 5 & 5 & 10 \\ \text { No } & 89 & 255 & 344\end{array}$

Mercado Extranjero

$\begin{array}{rrrr}\text { Sí } & 1 & 16 & 17 \\ \text { No } & 93 & 244 & 337\end{array}$

Sector Económico

$\begin{array}{llll}\text { Primario } & 9 & 14 & 23\end{array}$

$\begin{array}{llll}\text { Secundario } & 15 & 54 & 69\end{array}$

$\begin{array}{llll}\text { Terciario } & 70 & 192 & 262\end{array}$

Fuente: Elaboración propia

\section{Análisis Multivariante}

Para determinar cuáles son los factores que influyen en la probabilidad de que una mujer ocupe cargos gerenciales, esta investigación hace uso de un modelo de regresión binaria tipo Logit. En este se utiliza como variable dependiente la variable categórica binomial Género del Gerente que toma el valor de 1 cuando el cargo de gerente es ocupado por una mujer y 0 cuando es ocupado por un hombre. Como variables de control se incluyen características tanto del gerente como de la empresa, las cuales permiten establecer estadísticamente qué factores incrementan o disminuyen la probabilidad de que una mujer ocupe el cargo gerencial en las Pymes de la ciudad de Cali.

El modelo Logit estimado es:

$$
\begin{aligned}
& P(Y=1 \mid \boldsymbol{X})=\boldsymbol{X} \boldsymbol{\beta}+\boldsymbol{\varepsilon}=\beta_{0}+\beta_{1} \text { Edad }+\beta_{2} \text { Estudios gerente }+\beta_{3} \text { Años empresa }+ \\
& \beta_{4} \text { Junta mujeres }+\beta_{5} \text { Junta directiva }+\beta_{6} \text { Participacion mujeres }+\beta_{7} \text { Ventas }+ \\
& \beta_{8} \text { Mercado extranjero }+\beta_{9} \text { Sector economico }+\beta_{10} R R H+\beta_{11} R R+\beta_{12} R S A+ \\
& \beta_{13} R P I+\varepsilon
\end{aligned}
$$


En donde

$\mathrm{Y}=1$, si la gerente es mujer, 0 si es hombre.

RRH = Rendimiento en Relaciones Humanas.

RR = Rendimiento Racional.

RSA = Rendimiento en Sistema Abierto.

RPI = Rendimiento en Procesos Internos.

Para facilitar la interpretación se reportan los coeficientes estimados del modelo Logit y los Odds ratio. Estos últimos miden la probabilidad de tener una mujer gerente $(\mathrm{Y}=1)$ en términos relativos a la probabilidad de tener un hombre como gerente en la empresa $(\mathrm{Y}=0)$. De esta manera un Odds ratio significativo y mayor (menor) a 1 refleja que ese factor se relaciona de manera positiva (negativa) con la probabilidad de que una mujer se desempeñe como gerente. En la Tabla 3 se reportan los resultados de la estimación.

\section{Tabla 3}

Resultados modelo Logit

Variables explicativas

Edad del gerente

$\begin{array}{ccc} & (0,0134) & (0,0126) \\ \text { Estudios del gerente } & 0,4613 & 1,5861 \\ \text { Años de la empresa } & (0,386) & (0,6371) \\ \text { Junta Mujeres } & 0,0283 * * & 1,0287 * * * \\ \text { Junta Directiva } & (0,0143) & (0,0150) \\ & 0,0275 * & 1,0279 * \\ \text { Participación Mujeres } & (0,00613) & (0,0057) \\ & 3,0363 * & 20,8288 * \\ \text { Log Ventas } & (0,8208) & (17,1436) \\ & 0,02817 * & 1,0285 * \\ & (0,0977) & (0,0110) \\ & -0,1574 & 0,8543 * * * \\ & & (0,08100)\end{array}$

Odds Ratio

$0,9756 * * *$

$-0,0246^{* * * *}$

ogit

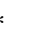


Mercado extranjero

$-1,7898^{* *}$

$(0,8039)$

Sectores

\begin{tabular}{|c|c|c|}
\hline Sector primario & $1,1153 * *$ & $3,0506 * *$ \\
\hline & $(0,5234)$ & $(1,6719)$ \\
\hline \multirow[t]{2}{*}{ Sector secundario } & $-0,04823 * * *$ & $0,9529 * * *$ \\
\hline & $(0,3833)$ & $(0,3716)$ \\
\hline \multirow[t]{2}{*}{ Rendimiento Relaciones Humanas } & $-0,5289 * *$ & $0,5544 * * *$ \\
\hline & $(0,2943)$ & $(0,1766)$ \\
\hline \multirow[t]{2}{*}{ Rendimiento Racional } & $-0,8581 *$ & $0,4239 *$ \\
\hline & $(0,2811)$ & $(0,1237)$ \\
\hline \multirow[t]{2}{*}{ Rendimiento Sistema abierto } & $0,8236 * *$ & $2,2788 * *$ \\
\hline & $(0,3242)$ & $(0,7239)$ \\
\hline \multirow[t]{2}{*}{ Rendimiento Procesos Internos } & $0,1654 * * *$ & $1,1799 * * *$ \\
\hline & $(0,3792)$ & $(0,4499)$ \\
\hline \multirow[t]{2}{*}{ Constante } & 3,0393 & 20,89076 \\
\hline & $(2,5167)$ & $(53,7202)$ \\
\hline Pseudo R2 & & 0,1998 \\
\hline Wald Chi2 & & 64,08 \\
\hline Prob $>$ chi 2 & & 0,0000 \\
\hline
\end{tabular}

Nota: Errores estándar entre paréntesis

$(*): \mathrm{p}<0.01 ;(* *): \mathrm{p}<0.05 ;(* * *): \mathrm{p}<0.1$

Fuente: Elaboración propia

Los resultados encontrados muestran que un incremento en los años de la empresa, la presencia de junta directiva, una mayor proporción de mujeres en la junta directiva, mayor participación de las mujeres, pertenecer al sector primario de la economía y presentar rendimiento en sistema abierto y procesos internos, son factores que tiene una relación positiva con la probabilidad de que una mujer sea la gerente de una Pyme. Es importante resaltar que el factor que más peso tiene a la hora de determinar la probabilidad de que una mujer sea gerente es la presencia de junta directiva. Estos resultados van en línea con los encontrado 
en la literatura en distintos países (Gupta \& Raman, 2014; Matsa et al., 2011; Ng \& Sears, 2017). Estos resultados también van de la mano con la trayectoria gerencial de las mujeres por lo que al ocupar cargos en juntas directivas promueven la inclusión de más mujeres en cargos gerenciales.

Por otra parte, la edad del gerente, la participación en el mercado extranjero, pertenecer al sector secundario, el rendimiento en relaciones humanas y el rendimiento racional tienen un impacto negativo en la probabilidad de que una mujer sea gerente de una organización. Además de esto, es importante resaltar que contrario a lo esperado, el nivel educativo no es estadísticamente significativo a pesar de que trabajos como el de Hurley \& Choudhary 2016, Hodigere \& Bilimoria 2015) resaltan su importancia (aunque estos estudios no se realizan para el caso de las Pymes). Por lo anterior, se recomienda a los estudios posteriores considerar no solo el nivel educativo, sino analizar las áreas del conocimiento de los gerentes con el fin de explicar de manera más detallada estos resultados.

El efecto negativo de la variable de internacionalización va en línea con los resultados encontrados por $\mathrm{Ng} \&$ Sears (2017) los cuales argumentan que empresas que sean foráneas o cuenten con presencia internacional prefieren contratar directivos de género masculino, ya que consideran que estos tienen mejores resultados en las negociaciones o están atadas por criterios de selección impuestos por otros países.

De igual modo, el efecto negativo encontrado en el sector secundario muestra la importancia que tienen factores de tipo macroeconómico en la capacidad de crecimiento laboral por parte de las mujeres, ya que además de encontrar barreras de tipo empresarial pueden llegarse a encontrar con barreras sectoriales dentro del país y fuera del mismo. Los resultados anteriores apuntan a tratar el problema de desarrollo profesional de las mujeres no solo bajo un enfoque micro al interior de la empresa, sino también tener en cuenta los aspectos macro de las dinámicas empresariales (Kirsch, 2017).

Asumiendo los resultados presentados anteriormente y las hipótesis presentadas al inicio del texto, podemos determinar la validez de las mismas. La Hipótesis 1 plantea que las mujeres con mayor nivel educativo tienen mayor probabilidad de ser gerentes, sin embargo, como mencionamos anteriormente, la variable no es estadísticamente significativa, por lo que en esta investigación los niveles educativos no tienen ninguna influencia en la probabilidad de que una mujer sea gerente. Por otra parte, de acuerdo con la Hipótesis 2, la edad de las mujeres tiene una relación positiva con la probabilidad de que estas lleguen a ser gerentes; en este caso la edad del gerente es una variable significativa y tiene un impacto negativo, de ahí que se rechaza la hipótesis y se establece que entre mayor sea la mujer disminuye la probabilidad de que llegue a ser gerente.

En cuanto a la edad de la empresa, la Hipótesis 3 propone que las empresas gestionadas por mujeres son más jóvenes, no obstante los resultados revelan que la relación es positiva, 
de ahí que se rechaza la hipótesis. En contraste, las hipótesis 4 y 5 evalúan la influencia que tiene la presencia de junta directiva y la composición de la misma en la probabilidad de que una mujer llegue a ser gerente; de acuerdo con los coeficientes, ambas variables son estadísticamente significativas por lo que se acepta la hipótesis de que en las empresas donde hay junta directiva se incrementa la probabilidad de que el gerente sea mujer; así mismo, en estas Pymes la presencia de mujeres en la junta directiva es mayor. En este sentido, la Hipótesis 6 plantea que las mujeres tienen una mayor participación en las empresas gestionadas por mujeres, la variable es estadísticamente significativa y de signo positivo, por lo que podemos decir que las gerentes mujeres prefieren que la mano de obra de sus empresas sea femenina.

Con respecto a la Hipótesis 7, la cual propone que las empresas que se encuentran en el sector terciario son gestionadas por mujeres, podemos decir que no existe evidencia suficiente para aceptar o rechazar dicha hipótesis, ya que al realizar la estimación esta variable es omitida por el modelo. En la Hipótesis 8 se plantea que el crecimiento de las ventas en empresas gestionadas por mujeres es menor; esta variable no es estadísticamente significativa, por lo que no es posible determinar si el crecimiento de las ventas tiene influencia en la probabilidad de que una mujer sea gerente. Por otro lado, considerando la Hipótesis 9 se puede afirmar que la participación en el mercado extranjero sí tiene influencia en la probabilidad de que una mujer sea gerente y esta relación es negativa. Adicionalmente, teniendo en cuenta los Odds Ratio se puede determinar si esta probabilidad es menor con respecto a la de los hombres; al analizar los resultados podemos concluir que es correcto afirmar que la probabilidad de participar en el mercado extranjero con respecto a la de los hombres es menor para las mujeres. Para finalizar, la Hipótesis 10 propone una relación inversa entre la probabilidad de que una mujer sea gerente y el rendimiento. Los resultados muestran que esta relación es correcta para el Rendimiento en Relaciones Humanas y el Rendimiento Racional, sin embargo, se rechaza esta hipótesis para el Rendimiento de Sistema Abierto y de Procesos Internos.

\section{Conclusiones}

Las variables que influyen en la presencia de una mujer en un cargo directo son diversas. Esta investigación se realiza con el fin de establecer qué variables afectan la probabilidad de que una mujer ocupe un cargo gerencial en las Pymes de Colombia. Los resultados muestran que la probabilidad de que una mujer sea gerente de una Pyme se encuentra influenciada principalmente por la edad del gerente, los años de la empresa, la presencia de junta directiva, la cantidad de mujeres presentes en la junta, la participación de las mujeres en las actividades productivas, las exportaciones, el sector y el rendimiento. Para evaluar la influencia de cada una de estas variables se realizó un modelo tipo Logit que permitió evaluar la probabilidad 
de que un evento ocurra; en este caso, la variable dependiente era la probabilidad de que una mujer sea gerente.

$\mathrm{Al}$ analizar detalladamente cada una de las variables y las hipótesis planteadas al inicio se encuentra que se rechazan las hipótesis relacionadas con el nivel educativo, la edad del gerente y los años de funcionamiento de la empresa. Por el contrario, se aceptan las hipótesis que consideran la presencia de junta directiva, la cantidad de mujeres presentes en la junta directiva, la participación de las mujeres y las exportaciones de la empresa. De acuerdo con esto, se puede afirmar que si la empresa tiene junta directiva y esta se encuentra conformada en una mayor parte por mujeres y adicionalmente hay una mayor participación de mujeres en la empresa y una baja participación de la Pyme en los mercados extranjeros, se incrementa la probabilidad que esta sea gerenciada por una mujer. Estos resultados permiten concluir que para que una mujer ocupe cargos gerenciales es necesario que ella también tenga mayor participación laboral y se olviden los roles anteriormente conocidos.

No obstante, es importante mencionar que variables como el sector y las ventas no fueron estadísticamente significativas: la primera variable solo se encuentra representada en el sector primario y secundario, ya que en el momento de la estimación la variable que consideraba el sector terciario fue omitida por falta de observaciones; con respecto a la segunda, se encuentra que para esta investigación el coeficiente no fue estadísticamente significativo, por lo que no es posible determinar si las ventas tienen una relación con la probabilidad de que una mujer sea gerente. En este sentido, la participación de la mujer ya está dada en todos los sectores de la economía y puede liderarlos plenamente. Así mismo, la relación de ventas con la mujer indica que no necesariamente se está vinculando a las mujeres a cargos estratégicos cuando la empresa está en crisis.

Es importante resaltar que en el rendimiento se consideraron 4 modelos: relaciones humanas, racional, procesos internos y sistema abierto. Cada uno de estos fue considerado como una variable independiente; por lo anterior, de acuerdo con los resultados el rendimiento en relaciones humanas y el racional tienen un impacto positivo en la probabilidad de que una mujer sea gerente, mientras que el rendimiento en procesos internos y de sistema abierto tienen un impacto negativo. En otras palabras, cuando las ventas, la participación del mercado, los niveles de rotación y ausentismo están bien, las mujeres pueden ocupar más fácilmente cargos gerenciales. Sin embargo, cuando la calidad del producto / servicio, los procesos operativos, la satisfacción de los clientes y la imagen de la empresa no están bien, hay pocas probabilidades de que una mujer ocupe estos cargos. Estos resultados pueden ser atribuidos a los roles que la mujer todavía ejerce en la sociedad y a las características que tradicionalmente se le atribuyen, como poca operatividad, organización y eficiencia, además de características humanas como amabilidad, cordialidad y relación con el empleado. Por este motivo, futuras investigaciones deberían realizar un análisis detallado de la influencia que 
tiene el rendimiento en procesos internos y sistema abierto en la probabilidad de ser gerente para evidenciar características propias del género.

Dentro de las limitaciones de esta investigación se encuentra la recolección de los datos, pues esta fue realizada con un cuestionario autoadministrado que el mismo gerente respondió. Futuras investigaciones pueden aplicar el cuestionario también a empleados para contrastar resultados. Otras investigaciones también pueden incluir variables relacionadas con la familia y condiciones personales para hacer un modelo más robusto tales como las de Cárdenas et al. (2014), Eagly y Carli (2007) y Eagly y Karau (2002) quienes incluyen número de hijos de los gerentes, conformación familiar y características de la personalidad.

Todos los resultados anteriores abren una puerta a la investigación de género. Sin embargo, hay que ser cautelosos al momento de implementar estos hallazgos en la práctica, ya que si no se tratan como se debe puede aumentar más las brechas de género. Ahora bien, este estudio lo que pretende a nivel práctico es que se aumente la presencia de mujeres ejecutivas. En primer lugar, las mujeres con mucha experiencia en el campo de los negocios y con empresas maduras tienen la posibilidad de ocupar cargos directivos, ello depende directamente de la eliminación de otros obstáculos sociales para lograrlo. En segundo lugar, aumentar la participación de mujeres en una empresa y en la junta directiva hace que las empleadas necesiten de una mujer que las represente y por eso las mujeres directivas pueden jugar un papel importante en este tipo de liderazgo. En tercer lugar, la diversidad de género se convierte en un tema transversal, ya que para llevar a las mujeres gerentes al nivel ejecutivo y de la junta directiva se debe crear espacios de participación de igualdad entre hombres y mujeres. Así, las empresas tendrán que cambiar las trayectorias de esas mujeres y permitirles recuperar el terreno perdido con promociones y ascensos en general para aumentar la participación directiva.

\section{Referencias}

Acar, F. P. (2015). Gender Differences in Promotions to Top Level Management Positions: An Examination of Glass Cliff in the IT Sector. Procedia - Social and Behavioral Sciences, 210, 223-230. https://doi.org/10.1016/j.sbspro.2015.11.362

Adams, R. B., \& Ferreira, D. (2008). Women in the boardroom and their impact on governance and performance. Journal of Financial Economics, 45, 291-309. https://doi. org/10.1016/j.jfineco.2008.10.007

Adams, R. B., \& Ferreira, D. (2009). Women in the boardroom and their impact on governance and performance. Journal of Financial Economics, 94(2), 291-309. https://doi. org/10.1016/j.jfineco.2008.10.007

Adams, S. M., Gupta, A., \& Leeth, J. D. (2009). Are female executives over-represented in precarious leadership positions? British Journal of Management, 20(1), 1-12. https://doi. org/10.1111/j.1467-8551.2007.00549.x 
Ali, J., \& Shabir, S. (2017). Does gender make a difference in business performance? Gender in Management: An International Journal, 32(3), 218-233. https://doi.org/10.1108/ GM-09-2016-0159

Araújo-Pinzón, P., Álvarez-Dardet, C., Ramón-Jerónimo, J. M., \& Flórez-López, R. (2017). Women and inter-organizational boundary spanning: A way into upper management? European Research on Management and Business Economics, 23(2), 70-81. https://doi. org/10.1016/j.iedeen.2016.11.001

Barnet-Verzat, C., \& Wolff, F.-C. (2008). Gender wage gap and the glass ceiling effect: a firm-level investigation. International Journal of Manpower, 29(6), 486-502. https://doi. org/10.1108/01437720810904185

Beldad, A., Hegner, S., \& Hoppen, J. (2016). The effect of virtual sales agent (VSA) gender - Product gender congruence on product advice credibility, trust in VSA and online vendor, and purchase intention. Computers in Human Behavior, 60, 62-72. https://doi. org/10.1016/j.chb.2016.02.046

Brady, D., Isaacs, K., Reeves, M., Burroway, R., \& Reynolds, M. (2011). Sector, size, stability, and scandal: Explaining the presence of female executives in $<$ IT $>$ Fortune $</$ IT $>$ 500 firms. Gender in Management: An International Journal, 26(1), 84-105. https://doi. org/10.1108/17542411111109327

Burgess, Z., \& Tharenou, P. (2002). Women Board Directors : Characteristics of the Few Board Directors : of the Few Characteristics panies. Journal of Business Ethics, 37(1), 39-49. https://doi.org/10.1023/A:1014726001155

Burin, M. (2008). Las "fronteras de cristal" en la carrera laboral de las mujeres. G??nero, subjetividad y globalización. Anuario de Psicologia, 39(1), 75-86.

Cámara de Comercio. (2014). Gerencia de desarrollo y competitividad. Apunte Economico, 11,2 .

Cárdenas, M. C., Eagly, A., Salgado, E., Goode, W., Heller, L. I., Jaúregui, K., Tunqui, R. C. (2014). Latin American female business executives: an interesting surprise. Gender in Management: An International Journal, 29(1), 2-24. https://doi.org/10.1108/GM-062013-0067

Centre for Social Development, Humanitarian Affairs, UNICEF, \& United Nations Development Fund for Women. (2015). The World's Women 2015: Trends and Statistics. United Nations.

Congreso de la República de Colombia. Ley 905 de 2004, 33 Uma ética para quantos? (2004). https://doi.org/10.1007/s13398-014-0173-7.2

Cook, A., \& Glass, C. (2014). Women and Top Leadership Positions: Towards an Institutional Analysis. Gender, Work and Organization, 21(1), 91-103. https://doi.org/10.1111/ gwao.12018

Di Ciano, M. (2010). Pymes - Rol en la Economía Nacional y características particulares de funcionamiento, (3), 1-16. Disponible en https://aduba.org.ar/wp-content/uploads/2016/07/Pymes.pdf y Consultado: 12/01/2018. 
Díaz, M. . C., \& Jiménez, J. J. . (2010). Recursos y resultados de las pequeñas empresas: nuevas perspectivas del efecto género. Cuadernos de Economía y Dirección de La Empresa, 13(42), 151-175. http://dx.doi.org/10.1016/S1138-5758(10)70006-2

Duarte, P. D. P., \& García, M. L. S. (2017). El Desempeño Exportador y la Innovación como una Estrategia de Crecimiento para la PYME en México. Revista Mexicana de Economía y Finanzas, 11(2), 21-38. Disponible en http://congreso.investiga.fca.unam.mx/docs/ xviii/docs/1.08.pdf y Consultado 15/02/2018.

Eagly, A., \& Carli, L. L. (2007). Through the labyrinth: The truth about how women become leaders. Harvard Business Press.

Eagly, A. H., \& Karau, S. J. (2002). Role Congruity Theory of Prejudice Toward Female Leaders. Psychological Review, 109(3), 573-598. https://doi.org/10.1037//0033295X.109.3.573

Eduardo, M., \& Poole, B. (2016). CEO age and gender : Subsequent market performance. Cogent Business \& Management, 3(1), 1-8. https://doi.org/10.1080/23311975.2016.1146389

Elsaid, E. (2015). Comparing Outgoing Female CEOs With Prior CEO Experience To Outgoing Female CEOs With No Prior CEO Experience. The Journal of Applied Business Research, 31(3), 809-820. https://doi.org/110.19030/jabr.v31i3.9219

Foro Económico Mundial. (2016). The Global Gender Gap Report. Disponible en http:// www3.weforum.org/docs/GGGR16/WEF_Global_Gender_Gap_Report_2016.pdf y Consultado el 10/05/2017.

Freeman, S. (1990). Managing lives: Corporate women and social change. University of Massachusetts Press.

García, C. D., \& Moreno, J. J. J. (2009). Recursos y resultados de las pequeñas empresas: nuevas perspectivas del efecto género. Cuadernos de Economía y Dirección de La Empresa, 42, 151-175. https://doi.org/10.1016/S1138-5758(10)70006-2

García, M., García Pérez de Lema, D., \& Madrid Guijarro, A. (2012). Caracterización del comportamiento de las Pymes según el género del gerente: un estudio empírico, 28(47), 37-52. https://doi.org/10.25100/cdea.v28i47.72

Glass, C., \& Cook, A. (2016). Leading at the top: Understanding women's challenges above the glass ceiling. Leadership Quarterly, 27(1), 51-63. https://doi.org/10.1016/j.leaqua.2015.09.003

Gupta, A., \& Raman, K. (2014). Board diversity and ceo selection. Journal of Financial Research, 37(4), 495-518. https://doi.org/10.1111/jfir.12044

Haslam, S. A., \& Ryan, M. K. (2008). The road to the glass cliff: Differences in the perceived suitability of men and women for leadership positions in succeeding and failing organizations. Leadership Quarterly, 19(5), 530-546. https://doi.org/10.1016/j.leaqua.2008.07.011

Hodigere, R., \& Bilimoria, D. (2015). Human capital and professional network effects on women's odds of corporate board directorships. Gender in Management: An International Journal, 30(7), 523-550. https://doi.org/10.1108/GM-07-2015-0063 
Hoogendoorn, S., Hessel, O., \& Mirjam, V. P. (2013). The impact of gender diversity on the performance of business teams : Evidence from a field experiment. Management Science, 59(7), 1514-1528. https://doi.org/10.2139/ssrn.1826024

Hurley, D., \& Choudhary, A. (2016). Factors influencing attainment of CEO position for women. Gender in Management: An International Journal, 31(4), 250-265. https://doi. org/10.1108/GM-01-2016-0004

Jayme, M., \& Sau, V. (2004). Psicología diferencias del sexo y el género: fundamentos. Barcelona: Icaria Editorial.

Kirsch, A. (2017). The gender composition of corporate boards: A review and research agenda. Leadership Quarterly, 29(2) 346-364. https://doi.org/10.1016/j.leaqua.2017.06.001

Lee, I. H., Paik, Y., \& Uygur, U. (2016). Does Gender Matter in the Export Performance of International New Ventures? Mediation Effects of Firm-specific and Country-specific Advantages. Journal of International Management, 22(4), 365-379. https://doi. org/10.1016/j.intman.2016.05.004

Matsa, D. A., Miller, A. R., \& Bertrand, M. (2011). Chipping away at the Glass Ceiling : Gender Spillovers in Corporate, 101(3), 635-39. Disponible en https://pubs aeaweb.org/ doi/pdfplus/10.1257/aer.101.3.635 y Consultado 25/08/2018

Meza Martínez, C.A. (2018). Discriminación laboral por género: una mirada desde el efecto techo de cristal. Equidad y Desarrollo, (32), 11-31. https://doi.org/10.19052/ed.5243

Ministerio de Comercio Industria y Turismo. (2012). Definición Tamaño Empresarial Micro, Pequeña, Mediana o Grande. Disponible en https://www.mincit.gov.co/prensa/noticias/ industria/gobierno-expide-nueva-clasificacion-de-empresas-a y Consultado 18/03/2017

Moreno-Gómez, J., \& Calleja-Blanco, J. (2018). The relationship between women's presence in corporate positions and firm performance. International Journal of Gender and Entrepreneurship, 10(1), 83-100. https://doi.org/10.1108/IJGE-10-2017-0071

$\mathrm{Ng}, \mathrm{E}$., \& Sears, G. J. (2017). The glass ceiling in context: the influence of CEO gender, recruitment practices and firm internationalisation on the representation of women in management. Human Resource Management Journal, 27(1), 133-151. https://doi. org/10.1111/1748-8583.12135

Oakley, J. G. (2000). Gender-based Barriers to Senior Management Positions: Understanding the Scarcity of Female CEOs. Journal of Business Ethics, 27(1994), 321-334. https:// doi.org/10.2307/25074386

Powell, G. N., \& Eddleston, K. (2011). Work family enrichment and entrepreneurial success: do female entrepreneurs benefit most? Academy of Management Proceedings, 1 , 1-16. Disponible en https://journals.aom.org/doi/pdf/10.5465/ambpp.2011.65869189 y consultado el 28/08/2018

Quinn, R. E., \& Rohrbaugh, J. (1983). A Spatial Model of Effectiveness Criteria : Towards a Competing Values Approach to Organizational Analysis. Management Science, 29(3), $363-377$. 
Ryan, M. K., \& Haslam, S. A. (2005). The glass cliff: Evidence that women are over-represented in precarious leadership positions. British Journal of Management, 16(2), 81-90. https://doi.org/10.1111/j.1467-8551.2005.00433.x

Smith, N., Smith, V., \& Verner, M. (2011). Why Are So Few Females Promoted into CEO and $\backslash n$ Vice-President Positions? ILR Review, 66(2), 380-408. Disponible en http://ftp. iza.org/dp5961.pdf y Consultada el 28/04/2018

Valencia, J. R. (2000). Gemeralidades de las pymes. In Adminsitración de pequeñas y medianas empresas (p. 357). México D.F.: Cengage Learning Editores.

Welch, C. L., Welch, D. E., \& Hewerdine, L. (2008). Gender and Export Behaviour : Evidence from Women-Owned Enterprises. Journal of Business Ethics, 83, 113-126. https:// doi.org/10.1007/s10551-007-9652-5

Williams, D. A. (2013). Gender and internationalization of SMEs. Journal of International Business Research, 12(1), 133-147. Disponible en https://www.abacademies.org/articles/jibrvol12no12013.pdf\#page=139 y Consultada el 28/04/2018

Withisuphakorn, P., \& Jiraporn, P. (2017). CEO age and CEO gender: Are female CEOs older than their male counterparts? Finance Research Letters, 22, 129-135. https://doi. org/10.1016/j.frl.2016.12.026 